Copyright (C) 2003 IEEE. Reprinted from

IEEE Transactions on Communications, 2003; 51 (2):161-165

This material is posted here with permission of the IEEE. Such permission of the IEEE does not in any way imply IEEE endorsement of any of the University of Adelaide's products or services. Internal or personal use of this material is permitted. However, permission to reprint/republish this material for advertising or promotional purposes or for creating new collective works for resale or redistribution must be obtained from the IEEE by writing to pubs-permissions@ieee.org.

By choosing to view this document, you agree to all provisions of the copyright laws protecting it. 


\title{
A New Bound and Algorithm for Star 16-QAM Carrier Phase Estimation
}

\author{
Feng Rice, Associate Member, IEEE, Mark Rice, and Bill Cowley, Member, IEEE
}

\begin{abstract}
The true Cramér-Rao lower bound (CRLB) is derived and evaluated for the estimation of carrier phase of Star 16-quadrature amplitude modulation (QAM) and can be simply applied to carrier frequency estimation. Different geometries are investigated by varying the ring ratio (RR). For signal-to-noise ratios (SNRs) between 6-15 dB, the CRLB with $R R=3$ is lower than that of Square 16-QAM. A modified phase estimator is presented, which closely follows the new CRLB. Investigation of symbol error performance in short packet length reveals Star 16-QAM to be superior to Square 16-QAM for $\mathrm{SNR}<13 \mathrm{~dB}$, which is a reasonable operating range for a coded system. Although Square 16-QAM and Star $R R=\mathbf{1 . 8}$ are optimum for a perfect receiver, when the effect of phase estimation is considered, we find $S \operatorname{tar} R R=3$ to be better for SNR below $10 \mathrm{~dB}$.
\end{abstract}

Index Terms-Cramér-Rao lower bounds (CRLB), frequency estimation, phase estimation, quadrature amplitude modulation (QAM), synchronization.

\section{INTRODUCTION}

$\mathbf{Q}$ UADRATURE amplitude modulation (QAM) techniques [2] are used to transmit $m$-bit symbols via a $2^{m}$ signal point constellation, distributed on a complex plane. By selecting large $m$, a highly bandwidth efficient modulation scheme can be designed.

Star 16-QAM [2] was proposed as it can be differential detected with noncoherent techniques. Differential detection is computationally simpler than coherent detection, however, it suffers a performance loss. Star QAM has the property that its peak-to-average power ratio is less than that for Square QAM. This means that Star QAM can operate at higher power (less backoff from saturation) in a practical radio transmitter. Therefore, there may be situations where Star QAM combined with coherent detector can be the preferred scheme.

The coherent detector requires phase knowledge which, in practice, must be estimated. The Cramér-Rao lower bound (CRLB) for the phase estimate of an unmodulated carrier wave (CW) is well known [3]. The modified CRLB (MCRB) [4]-[6] and the asymptotic CRLB (ACRB) [7] are good approximations for the true bound for $M$-ary phase-shift keying (MPSK)/QAM modulated signals at higher signal-to-noise ratios (SNRs).

Paper approved by R. Reggiannini, the Editor for Synchronization and Wireless Applications of the IEEE Communications Society. Manuscript received October 17, 2001; revised April 19, 2002 and July 3, 2002. This work was supported by the Cooperative Research Center for Sensor Signal and Information Processing and the Cooperative Research Center Satellite Systems.

F. Rice is with the Cooperative Research Center for Sensor Signal and Information Processing (CSSIP), University of Adelaide, Adelaide, SA 5005, Australia (e-mail: feng@cssip.edu.au).

M. Rice is with DSpace Pty. Ltd, Mawson Lakes, SA 5095, Australia (e-mail: mark@dspace.com.au).

B. Cowley is with the Institute for Telecommunications Research, University of South Australia, Mawson Lakes, SA 5095, Australia (e-mail: bill.cowley@unisa.edu.au).

Digital Object Identifier 10.1109/TCOMM.2003.809235
However, they depart significantly from the true CRLB at low SNR. Recently, the true CRLBs for Square QAM was derived and evaluated [1].

This paper is concerned with evaluating the CRLBs for Star 16-QAM and a modified phase estimator. We assume a static flat channel with no knowledge of the transmitted sequence, i.e., preamble or pilot symbols. Several authors have suggested phase estimators for this scenario [8]-[11]. Without knowledge of the true CRLB, it is impossible to know how far these techniques are from the fundamental performance limits.

In Section II, we derive the Star 16-QAM CRLB expression and evaluate it using a numerical approach. A comparison of CRLBs for CW, binary phase-shift keying (BPSK), quaternary phase-shift keying (QPSK) [12], 8-phase-shift keying (PSK), Star 16-QAM, and Square 16-QAM [1] is given. It is shown that the ratio $F\left(N_{o} / 2 E_{s}\right)$ of CRLB for a modulated signal to the CRLB for CW is the same for phase estimation and frequency estimation [1]. $F\left(N_{o} / 2 E_{s}\right)$ is particularly useful to designers in determining the limiting performance of synchronization circuits for coherent receivers for an arbitrary observation interval.

Section III presents a two-stage conjugate phase estimator for Star 16-QAM. This algorithm uses a non-decision-directed approach first, then a decision-directed approach. The simulation results are compared to the CRLBs evaluated in Section II. The performance of the new algorithm follows the new CRLB clearly at moderate SNR and converge to the bound at high SNR.

Section IV discusses the CRLBs for Star 16-QAM constellations with different inner to outer ring ratios (RRs). The probabilities of symbol error with various RRs for both a perfect receiver and optimum receiver in an additive white Gaussian noise (AWGN) channel also are presented. The perfect receiver is taken to be the receiver with perfect phase and frequency knowledge; whereas, the optimum receiver has phase (and frequency) estimate(s) with variance(s) equal to the (respective) CRLBs.

Section IV also investigates the effect of Star 16-QAM RR on symbol error rate. The RR effect on error rate is determined by considering an optimum receiver, i.e., with the phase estimation matching the CRLB. It is found that with an optimum receiver at SNR below $10 \mathrm{~dB}$, an RR of three gives better performance than other RRs, including RR $=1.8$ and Square 16-QAM. Brief conclusions are presented in Section V.

\section{TRUE CRLBS FOR STAR 16-QAM PHASE AND FREQUENCY ESTIMATION}

The Star 16-QAM has two level amplitudes, $R_{1}$ and $R_{2}$, that is, two circles around the origin. Each circle has eight phase points on the ring. Fig. 1 shows Star 16-QAM constellation with $R_{2} / R_{1}=3$. The unit average energy constellation set $\mathbf{C}=$ $(1 / \sqrt{10})\{ \pm \sqrt{2}, \pm \sqrt{18}, \pm j \sqrt{2}, \pm j \sqrt{18}, \pm 1 \pm j, \pm 3 \pm j 3\}$. 


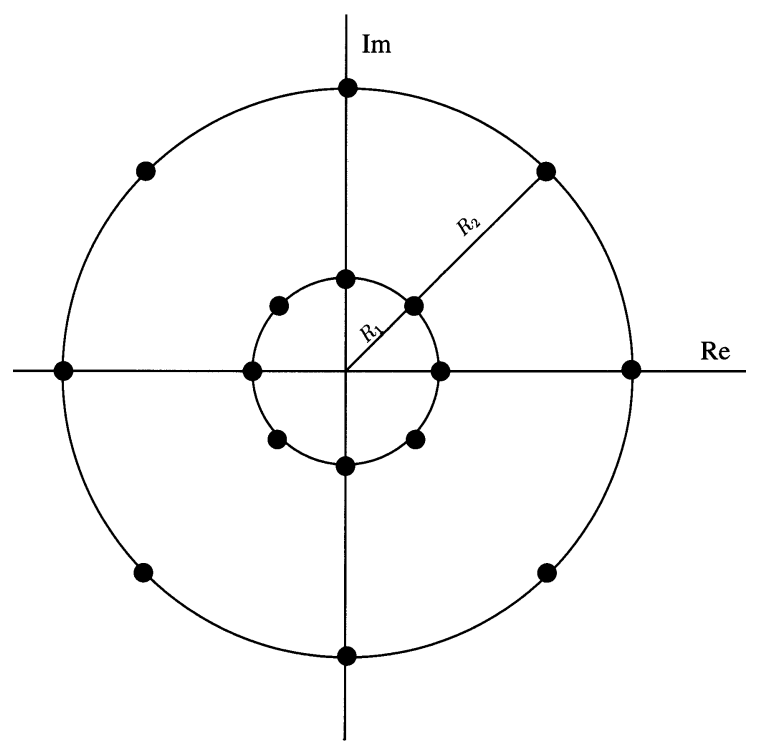

Fig. 1. Star 16-QAM constellation.

\section{A. Phase CRLB for Star 16-QAM}

We initially assume that a received QAM square constellation signal has an unknown fixed phase offset $\phi$, and that the signal has been ideally filtered and sampled at the optimum sampling instant. In this case, the received samples are

$$
x_{k}=a_{k} e^{j \phi}+w_{k}, \quad k=0,1, \ldots, N-1
$$

where $a_{k}$ are the transmitted symbols of Star 16-QAM constellation $\mathbf{C}$ of unit average energy. $w_{k}$ is the $k$ th noise sample whose real and imaginary parts are independent zero-mean Gaussian random variables, each with variance $\sigma^{2}$, and the $w_{k}$ 's are mutually independent.

We will use the convention that $p_{X}(x)$ is the probability density function (pdf) for a random variable $X$ and use boldface symbols to denote vectors; where the meaning is clear, we will drop the subscript.
The CRLB on the variances of an unbiased estimator of $\phi$, namely, $\hat{\phi}$ for a sequence of $N$ symbols, is given by [13]

$$
\operatorname{CRLB}(\hat{\phi})=\frac{1}{-E\left[\frac{\partial^{2} \ln p(\mathbf{X} \mid \phi)}{\partial \phi^{2}}\right]}
$$

where $\mathbf{X}=\left(X_{0} X_{1} \ldots X_{N-1}\right)$ and $E[\cdot]$ denotes statistical expectation with respect to the pdf $p_{\mathbf{X}}(\mathbf{x} \mid \phi)$.

The pdf of Star 16-QAM is given by can be derived as follows:

$$
\begin{aligned}
p(\mathbf{x} \mid \phi)= & \prod_{k} \frac{e^{-\left|x_{k}\right|^{2} / 2 \sigma^{2}}}{16 \pi \sigma^{2}} \sum_{b=R_{1}, R_{2} r=0,2, \pm 1} \sum e^{-b^{2} / 2 \sigma^{2}} \\
& \cosh \left(\Re\left(\frac{\sqrt{2} b^{2}}{\sigma^{2}} b x_{k} e^{-j(\phi-r \pi / 4)}\right)\right)
\end{aligned}
$$

where $\Re$ and $\Im$ stand for real and imaginary parts.

Taking the logarithm and retaining the terms dependent on $\phi$, we obtain the corresponding log-likelihood function. Then take the second partial derivative with respect to $\phi$, as shown in (4) at the bottom of the page, where $\Lambda\left(x_{k}\right)$ is defined by the three terms within the large curly brackets.

The expectation of the second derivative is [1], [14]

$$
E\left[\frac{\partial^{2} \ln p(\mathbf{x} \mid \phi)}{\partial \phi^{2}}\right]=\frac{N}{\sigma^{2}} E\left[\Lambda\left(x_{k}\right)\right]
$$

Now define

$$
F\left(\sigma^{2}\right)=-E\left[\Lambda\left(x_{k}\right)\right]
$$

Thus, from (2) and (5), we obtain

$$
\begin{aligned}
\operatorname{CRLB}(\hat{\phi}) & =\frac{\sigma^{2}}{N} \frac{1}{F\left(\sigma^{2}\right)} \\
& =\frac{1}{2 N \frac{E s}{N o}} \frac{1}{F\left(\frac{N_{o}}{2 E s}\right)} .
\end{aligned}
$$

We note that $1 / 2 N(E s / N o$ ) in (7) corresponds to the CRLB for estimation of CW phase over $N$ symbol intervals.

$$
\begin{aligned}
\frac{\partial^{2} \ln p(\mathbf{x} \mid \phi)}{\partial \phi^{2}}= & \frac{1}{\sigma^{2}} \sum_{k}\left\{\frac{-\frac{1}{\sigma^{2}}\left[\sum_{b=R_{1}, R_{2}} \sum_{r=0,2, \pm 1} e^{-\left(b^{2} / 2 \sigma^{2}\right)} \sinh \left(\frac{\sqrt{2 b^{2}}}{\sigma^{2}} \Re\left(b x_{k} e^{-j(\phi-r \pi / 4)}\right)\right) \Im\left(b x_{k} e^{-j(\phi-r \pi / 4)}\right)\right]^{2}}{\left[\sum_{b=R_{1}, R_{2}} \sum_{r=0,2, \pm 1} e^{-\left(b^{2} / 2 \sigma^{2}\right)} \cosh \left(\frac{\sqrt{2 b^{2}}}{\sigma^{2}} \Re\left(b x_{k} e^{-j(\phi-r \pi / 4)}\right)\right)\right]^{2}}\right. \\
& +\frac{-\frac{1}{\sigma^{2}} \sum_{b=R_{1}, R_{2}} \sum_{r=0,2, \pm 1} e^{-b^{2} / 2 \sigma^{2}} \cosh \left(\frac{\sqrt{2 b^{2}}}{\sigma^{2}} \Re\left(b x_{k} e^{-j(\phi-r \pi / 4)}\right)\right)\left[\Im\left(b x_{k} e^{-j(\phi-r \pi / 4)}\right)\right]^{2}}{\sum_{b=R_{1}, R_{2}} \sum_{r=0,2, \pm 1} e^{-\left(b^{2} / 2 \sigma^{2}\right)} \cosh \left(\frac{\sqrt{2 b^{2}}}{\sigma^{2}} \Re\left(b x_{k} e^{-j(\phi-r \pi / 4)}\right)\right)} \\
& -\frac{\sum_{b=R_{1}, R_{2}} \sum_{r=0,2, \pm 1} e^{-\left(b^{2} / 2 \sigma^{2}\right)} \sinh \left(\frac{\sqrt{2 b^{2}}}{\sigma^{2}} \Re\left(b x_{k} e^{-j(\phi-r \pi / 4)}\right)\right) \Re\left(b x_{k} e^{-j(\phi-r \pi / 4)}\right)}{\sum_{b=R_{1}, R_{2}} \sum_{r=0,2, \pm 1} e^{-\left(b^{2} / 2 \sigma^{2}\right)} \cosh \left(\frac{\sqrt{2 b^{2}}}{\sigma^{2}} \Re\left(b x_{k} e^{-j(\phi-r \pi / 4)}\right)\right)} \\
= & \frac{1}{\sigma^{2}} \sum_{k} \Lambda\left(x_{k}\right)
\end{aligned}
$$




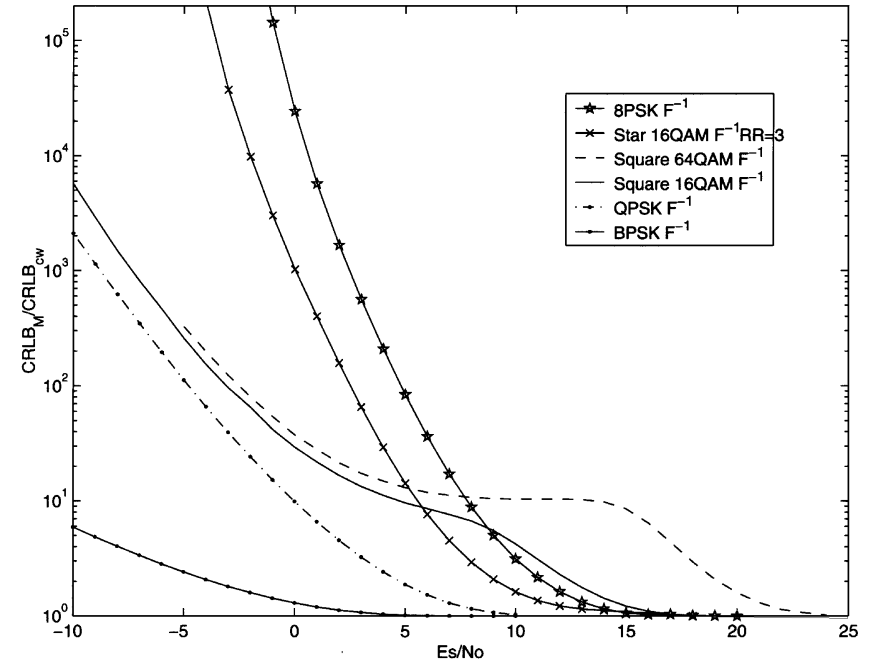

Fig. 2. Ratios $F_{m}^{-1}$ of CRLB for various modulations to CRLB for Carrier.

$F^{-1}\left(N_{o} / 2 E s\right)$ is, therefore, the ratio of the CRLB for random QAM signals to the CRLB for CW of the same power. Although an analytical solution for $F\left(N_{o} / 2 E s\right)$ is not feasible, we can evaluate it numerically.

The new result for Star 16-QAM with RR $=3$ is compared with the results from [1] in Fig. 2. The ratios $F^{-1}$ eventually converge to one as SNR increases, i.e., the CRLBs converge to the CRLB of CW. As expected, the more spectrally efficient modulations require a higher SNR to approach the CW CRLB. At low SNR, the simpler signal set has a lower CRLB. At SNR 6-15 dB, the CRLB with $\mathrm{RR}=3$ is lower than that of Square 16-QAM.

It may be observed that the Square 16-QAM and 64-QAM bounds converge at low SNRs. Once the symbols become indistinguishable due to noise, the shape of the constellation determines the CRLB, rather than the number of elements in the signal set. This also provides some explanation of the much worse performance of 8-PSK and Star 16-QAM. As might be expected, at $E_{s} / N_{o}$ below eight decibels, the circular shape of the constellation makes phase estimation difficult. In contrast, at higher SNRs, for all signal sets considered, it is the minimum distances between members of the set which determines where each bound departs from the $\mathrm{CW}$ bound.

Note that the ratios of $F\left(N_{o} / 2 E s\right)$ also apply to the CRLB for frequency estimation and joint phase and frequency estimation [1].

\section{Modified Two-Stage Phase Estimator}

To verify the new CRLBs, we have developed a modified phase estimator based on the two-stage conjugate algorithm in [1]. We use the RR 1.8 since it is the optimum RR for moderate to high SNR (Section V), and $a_{k}$ are the transmitted symbols of Star 16-QAM constellation

$$
\begin{aligned}
\mathbf{C} \in\left\{ \pm 2.17, \pm j 2.17, \pm 2.17 e^{ \pm j(\pi / 4)}\right. & \\
& \left. \pm 3.91, \pm j 3.91, \pm 3.91 e^{ \pm j(\pi / 4)}\right\}
\end{aligned}
$$

with average symbol energy 10 .
The signals are processed using a nondata-aided technique to produce an initial phase estimate. A Viterbi and Viterbi type of phase estimator (VVPE) [15] is employed. The estimated coarse phase $\hat{\phi}_{1}$ is

$$
\hat{\phi}_{1}=\frac{1}{8} \tan ^{-1} \frac{\sum_{k} \Im\left(\frac{x_{k}^{8}}{\left|x_{k}\right|^{7}}\right)}{\sum_{k} \Re\left(\frac{x_{k}^{8}}{\left|x_{k}\right|^{7}}\right)} .
$$

The phase is corrected for received signals $x_{k}$ using $\hat{\phi}_{1}$

$$
x_{k}^{\prime}=x_{k} e^{-j \hat{\phi}_{1}} .
$$

The next step is to estimate the transmitted symbols by demodulating the phase-corrected signals $x_{k}^{\prime}$ and choosing corresponding symbols $\hat{a}_{k}$ based on decision regions. The phase-corrected signals $x_{k}^{\prime}$ are then multiplied with the conjugate of the estimated transmitted signals to give

$$
y_{k}=x_{k}^{\prime} \cdot \hat{a}_{k}^{*} .
$$

The residual phase of $y_{k}$ can be calculated

$$
\hat{\phi}_{2}=\tan ^{-1} \sum_{k} \frac{\Im\left(y_{k}\right)}{\Re\left(y_{k}\right)} .
$$

The estimated phase offset is the sum of $\hat{\phi}_{1}$ and $\hat{\phi}_{2}$.

The phase estimate can be further refined by iterating the approach to generate $\phi$. This reduces the probability of error for the received signal $\hat{a}_{k}$ by successively using an improved phase estimate. For the case of a coded signal, the probability of symbol error can be significantly reduced by decoding the signal. This can improve the overall phase estimator performance and system error rate.

In order to determine performance, the phase estimator was simulated using a Monte Carlo technique. The Star 16-QAM signal was generated from a pseudorandom data source. The signal was then shifted by multiplying $e^{j \phi}$, where $\phi$ is a static phase offset taken from a random uniform distribution from $[-(\pi / 4),(\pi / 4)]$ (the boundaries of rotational symmetry for this constellation). Calibrated zero mean, complex Gaussian noise with variance $2 \sigma_{n}^{2}$ is added to the signal to generate $x_{k}$.

Fig. 3 shows simulation results with the observation interval $N=20$ and the new CRLBs. The vertical scale indicates standard deviation of the phase error in radians, and the horizontal scale shows SNR. In the simulation, 10000 packets of $N$ random Star 16-QAM symbols were generated to produce small measurement errors. The crossed line shown in the figure is the coarse estimator $\left(\hat{\phi}_{1}\right)$ and the dashed line is the two-stage phase estimator. The solid line represents the phase CRLB for Star 16-QAM, and the "o-" line is the phase CRLB for CW.

The results indicate that the new estimator follows the Star 16-QAM CRLB curve. The phase variance of the estimator is close to the Star 16-QAM CRLB at moderate SNR and approaches it at high SNR. There is a fraction of a decibel difference between the CRLB and the estimator in the range of $14 \mathrm{~dB} \leq \mathrm{SNR} \leq 16 \mathrm{~dB}$ for $N=20$. The performance gap reduces as $N$ increases. 


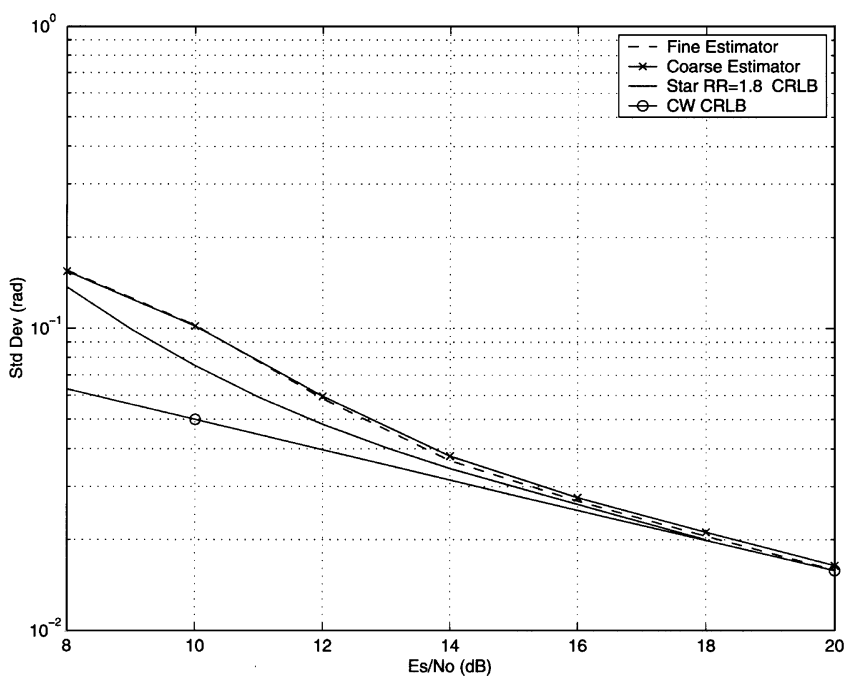

Fig. 3. Phase estimator performance of the new algorithm, $N=20$.

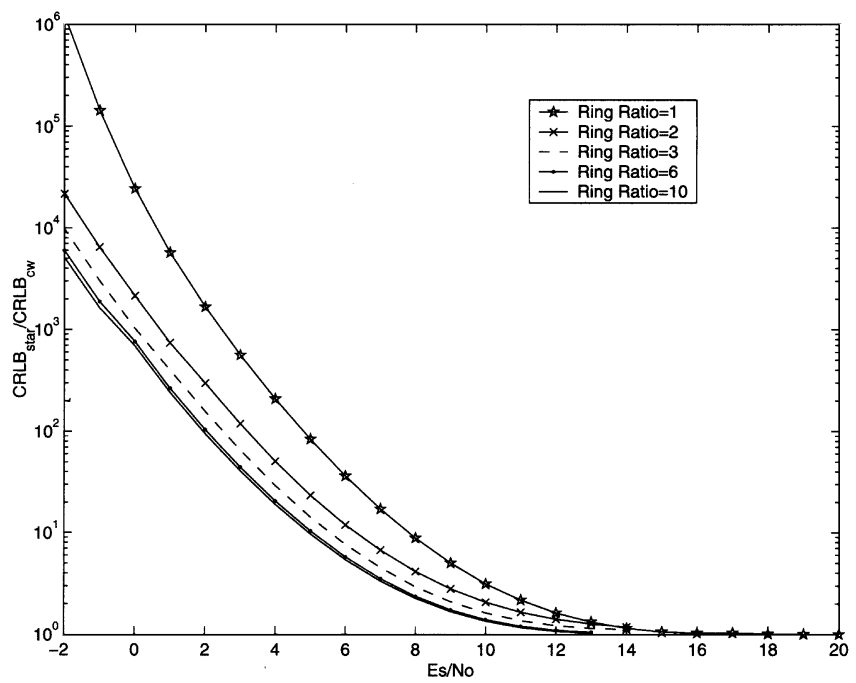

Fig. 4. Ratios $F_{\text {Star }}^{-1}$ of CRLB for various RR to CRLB for carrier.

\section{EFFECT OF RR ON ERROR RATE}

The Star 16-QAM RR is defined by [2] $\mathrm{RR}=R_{2} / R_{1}$. Under the constraint of a constant average symbol power equal to one, we obtain

$$
R_{1}^{2}=\frac{2}{1+\mathrm{RR}^{2}}, \quad R_{2}^{2}=\frac{2 \mathrm{RR}^{2}}{1+\mathrm{RR}^{2}} .
$$

We have presented CRLBs for RR $=3$ for AWGN channels as above. We can easily prove that the CRLBs for the $\mathrm{RR}=1 \mathrm{Star}$ 16-QAM is the same as the CRLB for 8-PSK [14, Ch. 5].

The numerical calculation was undertaken to compute $F\left(N_{o} / 2 E_{s}\right)$ with various RRs. The results are plotted in Fig. 4. At high SNR, all CRLBs converge to the CW CRLB, i.e., there is variance equality at high SNR.

As RR increases, the inner ring constellation points are compressed and become more susceptible to noise. How does this affect the probability of symbol error?

The receiver error rate is affected by both the signal constellation and the phase estimator perfomance. For a receiver with

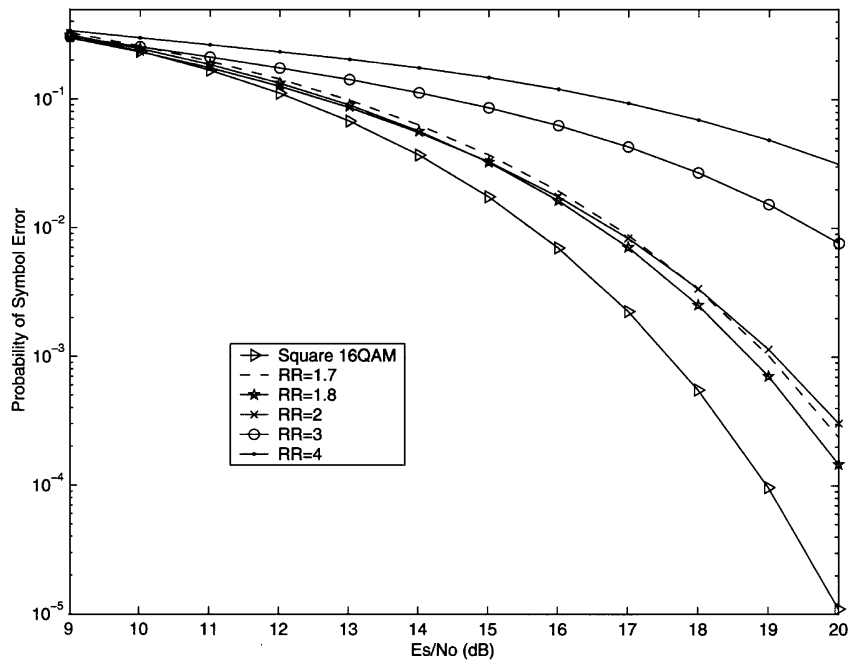

Fig. 5. Probability of symbol error for 16-QAM with perfect receiver in an AWGN channel.

phase offset of $\phi$ and RRs of RR, the symbol error rate can be derived from [16, Ch. 5], [17]

$$
\begin{aligned}
p_{s}(\phi) \simeq & \frac{1}{2}\left[\operatorname{erfc}\left(\frac{2 \sin ^{2}\left(\frac{\pi}{8}+\phi\right)}{1+\mathrm{RR}^{2}} \cdot \frac{E_{s}}{N_{o}}\right)\right. \\
& +\operatorname{erfc}\left(\frac{(\mathrm{RR}-1)^{2}}{2\left(1+\mathrm{RR}^{2}\right)} \cdot \frac{E_{s}}{N_{o}}\right) \\
& \left.+\operatorname{erfc}\left(\frac{2 \mathrm{RR}^{2} \sin ^{2}\left(\frac{\pi}{8}+\phi\right)}{1+\mathrm{RR}^{2}} \cdot \frac{E_{s}}{N_{o}}\right)\right] .
\end{aligned}
$$

Fig. 5 presents the probability of symbol error for the different RRs based on (12) for $\phi=0$. It shows that 1.8 is the optimum RR for Star 16-QAM. At low SNR, the symbol error rates for Star and Square 16-QAM are very close.

We can determine the effect of random phase error using [18, Ch. 9]

$$
p_{s}=\int_{-\infty}^{\infty} p(\phi) p_{s}(\phi) d \phi
$$

where $p(\phi)$ is the phase error pdf and $p_{s}(\phi)$ is the conditional probability of the symbol error. The Tikhonov pdf [16, eq. (6.77), Ch. 6] can be used to model the statistical distribution of the phase error. At high SNR, it approaches a Gaussian distribution. We calculated the performance of an optimum receiver assuming that the phase estimator variance has achieved the CRLB. The observation interval is chosen to be 20 symbols. The numerical integration of (13) has been computed assuming a Gaussian distribution (which is accurate above $10 \mathrm{~dB}$ ) and the performance loss relative to a perfect receiver with $R R$ $=1.8$ is presented in Fig. 6. Simulated results using the phase estimator from Section III and the perfect receivers for Star RR $=3$ and Square 16-QAM were also plotted for comparison. It is interesting to note that the simulated performance losses crossover around $10 \mathrm{~dB}$, which shows the $\mathrm{RR}=3$ performance to be better than the RR $=1.8$ and Square 16-QAM receiver at low SNR. From the simulated results, Star RR $=1.8$ performance loss is less than Square in the $8-13 \mathrm{~dB}$ range. 


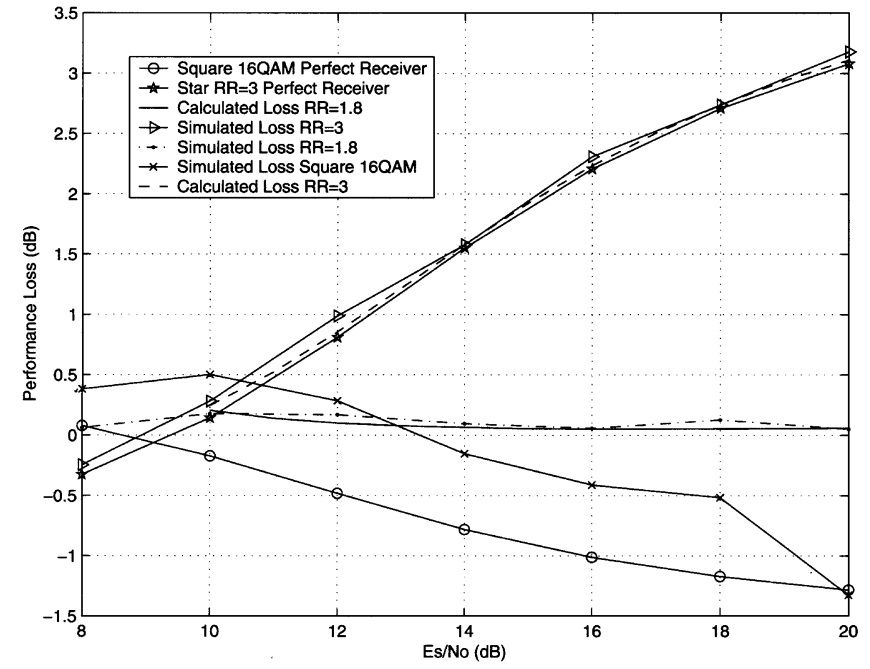

Fig. 6. Symbol error degradation for perfect, optimum, and simulated receivers $(N=20)$ relative to $\mathrm{RR}=1.8$ perfect $S$ tar 16 -QAM receiver.

For coded systems, the low SNR performance may be more important than at high SNR.

From Fig. 4, the phase estimation error decreases with increasing $\mathrm{RR}$ in the lower SNR range, and as a result, the RR $=3$ loss is less than the $\mathrm{RR}=1.8$ at $\mathrm{SNR} \leq 10 \mathrm{~dB}$. At high $\mathrm{SNR}$, the symbol error rate for $\mathrm{RR}=3$ is much higher than the one for $\mathrm{RR}=1.8$ (Fig. 5). This explains the bigger performance loss at moderate and high SNR in Fig. 6.

\section{CONCLUSION}

New CRLBs for carrier phase estimation of Star 16-QAM have been derived and evaluated. $F\left(N_{0} / 2 E_{s}\right)$ can also be applied to carrier frequency estimation as in [1]. At RR $=1$ the CRLBs are equivalent to that of 8-PSK. A modified phase estimator was developed, and the simulated results are presented which follow the CRLB closely. The RR effect on error rate was determined by considering an optimum receiver, i.e., with the phase estimation matching the CRLB. It is found that with an optimum receiver at SNR below $10 \mathrm{~dB}, \mathrm{RR}=3$ gives better performance than other RRs, including $\mathrm{RR}=1.8$ and Square 16-QAM. In the range of SNR from 8-13 dB where coded 16-QAM systems often operate, Star 16-QAM symbol error performance is better than that of Square 16-QAM for a small $N$.
For large $N$, Square 16-QAM has a smaller symbol error rate than Star 16-QAM at moderate to high SNR. The approach described of calculating the CRLB and using it to determine optimum receiver performance can be used to design good signal constellations.

\section{REFERENCES}

[1] F. Rice, B. Cowley, B. Moran, and M. Rice, "Cramér-Rao lower bounds for QAM phase and frequency estimation," IEEE Trans. Commun., vol. 49, pp. 1582-1591, Sept. 2001.

[2] W. T. Webb and L. Hanzo, Modern Quadrature Amplitude Modulation Principles and Applications for Fixed and Wireless Communications. Piscataway, NJ: IEEE Press, 1994.

[3] D. C. Rife and R. R. Boorstyn, "Single-tone parameter estimation from discrete-time observations," IEEE Trans. Inform. Theory, vol. IT-20, pp. 591-598, Sept. 1974.

[4] A. D'Andrea, U. Mengali, and R. Reggiannini, "The modified Cramer-Rao bound and its application to synchronization problems," IEEE Trans. Commun., vol. 42, pp. 1391-1399, Feb.-Apr. 1994.

[5] F. Gini, R. Reggiannini, and U. Mengali, "The modified Cramer-Rao bound in vector parameter estimation," IEEE Trans. Commun., vol. 46, pp. 52-60, Jan. 1998.

[6] U. Mengali and A. D'Andrea, Synchronization Techniques for Digital Receivers. New York: Plenum, 1997.

[7] M. Moeneclaey, "On the true and the modified Cramer-Rao bounds for the estimation of a scalar parameter in the presence of nuisance parameters," IEEE Trans. Commun., vol. 46, pp. 1536-1544, Nov. 1998.

[8] W. S. Yuan and C. N. Georghiades, "Rapid carrier acquisition from baud-rate samples," IEEE Trans. Commun., vol. 47, pp. 631-641, Apr. 1999.

[9] M. Moeneclaey and G. Jonghe, "ML-oriented NDA carrier synchronization for general rotationally symmetric signal constellations," IEEE Trans. Commun., vol. 42, pp. 2531-2533, Aug. 1994.

[10] C. Georghiades, "Blind carrier phase acquisition for QAM constellations," IEEE Trans. Commun., vol. 45, pp. 1477-1486, Nov. 1997.

[11] M. Luise and S. Pupolin, Broadband Wireless Communications. London, U. K.: Springer-Verlag, 1998.

[12] W. Cowley, "Phase and frequency estimation for PSK packets: Bounds and algorithms," IEEE Trans. Commun., vol. 44, pp. 1-3, Jan. 1996.

[13] S. Kay, Fundamentals of Statistical Signal Processing: Estimation Theory. Englewood Cliffs, NJ: Prentice-Hall, 1993.

[14] F. Rice, "Bounds and algorithms for carrier frequency and phase estimation," Ph.D. dissertation, Univ. South Australia, Mawson Lakes, Australia, 2001.

[15] A. J. Viterbi and A. M. Viterbi, "Nonlinear estimation of PSK-modulated carrier phase with application to burst digital transmission," IEEE Trans. Inform. Theory, vol. IT-29, pp. 543-551, July 1983.

[16] S. Benedetto, E. Biglieri, and V. Castellani, Digital Transmission Theory. Englewood Cliffs, NJ: Prentice-Hall, 1987.

[17] Y. C. Chow, A. R. Nix, and R. P. McGeehan, "Analysis of 16-APSK modulation in AWGN and Rayleigh fading channels," Electron. Lett., vol. 28, pp. 1608-1610, Aug. 1992.

[18] J. J. Stiffler, Theory of Synchronous Communications. Englewood Cliffs, NJ: Prentice-Hall, 1971. 